\title{
Enhancing memory and imagination improves problem solving among individuals with depression
}

\author{
Craig P. McFarland ${ }^{1} \cdot$ Mark Primosch $^{1} \cdot$ Chelsey M. Maxson $^{1} \cdot$ Brandon T. Stewart $^{1}$
}

Published online: 12 April 2017

(C) Psychonomic Society, Inc. 2017

\begin{abstract}
Recent work has revealed links between memory, imagination, and problem solving, and suggests that increasing access to detailed memories can lead to improved imagination and problem-solving performance. Depression is often associated with overgeneral memory and imagination, along with problem-solving deficits. In this study, we tested the hypothesis that an interview designed to elicit detailed recollections would enhance imagination and problem solving among both depressed and nondepressed participants. In a withinsubjects design, participants completed a control interview or an episodic specificity induction prior to completing memory, imagination, and problem-solving tasks. Results revealed that compared to the control interview, the episodic specificity induction fostered increased detail generation in memory and imagination and more relevant steps on the problem-solving task among depressed and nondepressed participants. This study builds on previous work by demonstrating that a brief interview can enhance problem solving among individuals with depression and supports the notion that episodic memory plays a key role in problem solving. It should be noted, however, that the results of the interview are relatively short-lived.
\end{abstract}

This research was supported by NIGMS Grant 5U54GM104944. We thank Tom Seekins for his guidance and mentorship, Solomon Harrar and Erin Semmens for statistical consultation, and Martina Haluzova, Sean Kavanaugh, Bianca Golling, James McFarland, and Mary McCarvel for testing and scoring. We also thank Laura Kirsch for feedback on previous drafts of this manuscript.

Craig P. McFarland

craig.mcfarland@umontana.edu

1 Department of Psychology, University of Montana, Skaggs 143, Missoula, MT 59812, USA
Keywords Depression · Memory · Imagination · Problem solving

Recent findings in psychological and cognitive neuroscience research have begun to illuminate the relations between memory and imagination. According to the constructive episodic simulation hypothesis (Schacter \& Addis, 2007), episodic memory and imagination both involve the retrieval of specific, detailed information about past experiences and thus rely on similar cognitive processes. In line with these ideas, episodic memory and imagination have been shown to recruit many of the same neural structures (Schacter, Addis, \& Buckner, 2008; Szpunar, 2010). These findings have contributed to theorizing that memory, in its most adaptive application, is future oriented (Klein, 2013). Furthermore, the links between memory and imagination raise the possibility that memory may play a role in a broad range of cognitive activities (Schacter, 2012).

The majority of research investigating the role that memory plays in other forms of cognition has focused on episodic memory, which is defined as memory for personally experienced past events that incorporate information pertaining to a specific time and place (Tulving, 1983, 1985). Semantic memory, on the other hand, can be defined as one's fund of knowledge that is derived from past experiences and includes factual information and vocabulary, as well as conceptual information about oneself (Klein \& Gangi, 2010). Although recent research has revealed that semantic memory plays a key role in other forms of cognition, including imagination (Abraham, 2014; Irish \& Piquet, 2013; Klein, 2013), the present article focuses on episodic memory.

Problem solving is one cognitive task that appears to rely upon the flexible, adaptive use of memory. Specifically, problem-solving tasks that involve open-ended or poorly defined problems involve the retrieval of episodic details from 
memory while at the same time requiring a future-directed orientation (see Abraham, 2014, for discussion of semantic memory contributions to similar cognitive processes). Like imagining, successful problem solving often requires making reference to one's past experiences (e.g., past successes or failures) and integrating the episodic details of those experiences in a way that leads to the development of novel, alternative solutions to a current problem. Support for this comes from studies showing a positive correlation between the number of relevant steps (defined as a step that could reasonably move a person toward problem solution) that older adults generate on a social problem-solving task and the number of details they recall about past personal events (Sheldon, McAndrews, \& Moscovitch, 2011). Similar findings have been reported among healthy young adults (Vandermorris, Sheldon, Winocur, \& Moscovitch, 2013). This research hints at fascinating possibilities for cognitive rehabilitation, including the potential of improving complex cognitive processes, such as problem solving, by enhancing access to episodic details in memory and imagination.

Further supporting the rehabilitation potential of this research, Madore and Schacter (2014) reported improved problem solving among healthy young and older adults following a brief intervention aimed at increasing details for recalled and imagined events. Participants completed two sessions during which they viewed one of two brief videos of common kitchen activities and were subsequently asked questions about the videos. In one session, participants were asked to provide their general impressions of the video. In the other session, participants were asked detailed questions about the video (e.g., what actions were performed, what objects were present) in an attempt to facilitate increased recall. Following the interviews, participants completed the Means-Ends Problem Solving task (MEPS; Platt \& Spivak, 1975), a social problem-solving task in which participants are presented with hypothetical problems and solutions to those problems, and are asked to generate steps that could lead the individual from problem onset to its solution. Participants were then presented a series of pictures and were asked to recall autobiographical memories or to imagine future scenarios related to the pictures. Young and older adults produced more relevant steps in the MEPS task after the detailed compared to the control interview. Additionally, both young and older adults produced significantly more details in the memory and imagination tasks following the intervention compared to the control interview, suggesting that increased access to details in recalled and imagined events may contribute to improved problem solving.

The effect that enhancing memory and imagination can have on problem solving may be especially important for individuals with depression. In addition to affective symptoms, depression is characterized by cognitive deficits, including reduced generation of details when recalling one's personal past (Hach, Tippett, \& Addis, 2014; Söderlund et al., 2014;
Williams et al., 2007) and imagining one's future (Holmes, Lange, Moulds, \& Steele, 2008). Moreover, recent research by Addis, Hach, and Tippett (2016) indicates that depression may differentially impact the generation of detailed future events relative to the recall of past events. Depression is also associated with problem-solving deficits, which have been linked to a reduced ability to generate specific details for past autobiographical events (Goddard, Dritschel, \& Burton, 1996; Raes et al., 2005; Williams et al., 2007), and may contribute to hopelessness (Cannon et al., 1999) and suicidal ideation (Williams, Barnhofer, Crane, \& Duggan, 2006). Improving problem solving among individuals with depression may lead to the development of alternative solutions to current problems, which in addition to reducing hopelessness and suicidal ideation may also contribute to an earlier resolution of a depressive episode or reduce the likelihood of recurrent depressive episodes. Given the links between depression and problem solving, and problem solving and suicidal ideation (Linda, Marroquín, \& Miranda, 2012), it is critical that effective interventions be identified.

The present study examined the possibility of improving problem solving among depressed individuals and used an intervention developed by Madore, Gaesser, and Schacter (2014) to facilitate detail generation in memory and imagination. Given previous findings of enhanced problem solving among healthy undergraduates and normally aging older adults, we predicted that individuals with depression would generate more details for recalled and imagined personal events following an episodic specificity induction compared to a general impressions interview. Most critically, we hypothesized that people with depression would evidence improved problem-solving performance following the episodic specificity induction.

\section{Method}

\section{Participants}

Forty-seven adults (ages18-50 years, $M=23.84, S D=8.75$ ) participated in this study. Participants were recruited via advertisements placed at the campus health center and psychology training clinic of a large Northwestern university, and via advertisements placed throughout the local community. The depressed group consisted of 23 participants (13 female; 10 male) who met DSM-IV criteria for a current major depressive episode. One depressed participant did not return for the second session, resulting in a total of 22 depressed participants. The nondepressed group consisted of 24 participants (16 female; seven male; one gender fluid) who endorsed few or no symptoms of depression. Two participants in the nondepressed group failed to follow instructions and were excluded from the analyses, resulting in a total of 22 nondepressed 
participants. Groups did not differ with regard to age or education $(p s>.83)$. Participants were paid for participating in the study. Ethical approval was obtained from the Internal Review Board.

\section{Experimental design}

Participants completed two sessions, approximately 1 week apart. To determine depression status, trained master's-level clinical psychology graduate students administered the mood module of the Structured Clinical Interview for DSM-IV-TR Axis-I Disorders (SCID-I; First, Spitzer, Gibbons, \& Williams, 2002) at the beginning of Session 1. Following this, in both sessions, participants (1) watched a brief video depicting two adults performing mundane kitchen activities, (2) answered questions about the videos in either a general impressions interview or an episodic specificity induction, (3) completed the MEPS (Platt \& Spivack, 1975), and (4) completed memory and imagination tasks. Different but similar experimental stimuli were used in each session (e.g., videos, problem-solving tasks, memory and imagination cues). The order of interview format (general impressions vs. episodic specificity induction) was counterbalanced across participants to control for possible order effects. The experimental design used in this study was taken from Madore and colleagues (Madore, Gaesser, \& Schacter, 2014; Madore \& Schacter, 2014), who provided all experimental materials used in this study (including videos, interview formats, and photographs). A power analysis based on the average effect sizes reported in Madore and Schacter (2014) revealed that a sample of 18 participants per group would be required to detect effects with power $>.80$ (two-tailed, $\alpha=.05, d=0.71$ ).

\section{Materials and procedure}

Interviews A general impressions interview served as the control condition. In this interview, participants were first asked to report their overall impressions of the video and then to answer a series of questions about their impressions or thoughts about the people, objects, or actions presented in the video. Finally, participants were asked whether the video reminded them of anything and when they thought the video was made.

Consistent with previous research (Madore \& Schacter, 2014), prior to the start of the episodic specificity induction, participants were told that they were an expert about the video they just watched, and were asked to recall, in as much detail as possible, details about the people, objects, and actions depicted in the video. Specific probing questions required participants to describe the appearance of the people and what they were wearing, the objects that were present and how they were arranged, and the actions that the people completed, in chronological order. This detailed retrieval was intended to foster the generation of more detailed personal memories and imagined events with the aim of investigating the role that those increased details may play in problem-solving performance. Both interviews required that participants think about the video. However, the episodic specificity induction required the participants to retrieve and discuss specific episodic details about the video rather than share general impressions of it.

Means-Ends Problem-Solving task (MEPS) After watching the video and completing the interview, participants completed the MEPS. All participants were presented with four different problems in each session. All problems include the statement of a problem (e.g., "Ms. P realizes that she has lost her watch.") and a successful resolution of that problem (e.g., "Ms. P is relieved to have found her watch."). Participants were asked to generate as many "ideal" steps to move from the statement of the problem to its successful solution. Participants were asked to type their responses on a computer and to generate as many steps as possible in as much detail as possible. Participants were allowed 5 minutes to generate steps for each problem in as much detail as possible. Participants completed the MEPS without any feedback or probing questions from the experimenter.

Memory and imagination tasks After completing the MEPS, participants completed the memory and imagination tasks. Participants were presented with eight pictures of generic scenes (e.g., airport, beach, carnival) and were asked to recall a personal experience (four pictures) and imagine a plausible future event (four pictures) related to the pictures. Participants were instructed to recall or imagine, from their own perspective, a specific, one-time event or day, rather than a frequently repeated or more extended event. Memories for a specific or one-time event are referred to as event-specific knowledge (Conway \& Pleydell-Pearce, 2000) and represent the level of memory at which depressed individuals generate fewer details than their nondepressed counterparts. Participants were asked to describe events that happened within the past 3 years or could occur within the next 3 years. As with the MEPS, participants were asked to type their responses and to include as much detail as possible, including information about people, objects, and actions. Participants were allowed 3 minutes to generate details for each recalled or imagined event. Pictures were blocked by task, and the order of tasks was counterbalanced across participants. Pictures were also randomized across tasks and participants. Participants completed the memory and imagination tasks without any feedback from the experimenter.

\section{Coding}

MEPS Typed responses to the MEPS were coded as relevant step, irrelevant step, or no step, in accordance with the scoring protocol described by Platt and Spivack (1975). A relevant 
step was one that could reasonably be expected to move the protagonist toward the problem solution. An irrelevant step is a step that moves the protagonist toward the solution of an unrelated problem. Finally, no step does not move the protagonist toward any goal or solution but often consists of commentary on the emotional state of the protagonist. In the MEPS example provided above, in which Ms. P lost her watch, a relevant step may be "she returned to her office to see if she had left it there." An example of an irrelevant step is "Ms. P purchased a new watch online." A no step could have been "Ms. P cried because of the sentimental value of the watch." For analytic purposes, we collapsed irrelevant steps and no steps into other steps, consistent with Madore and Schacter (2014).

Memory and imagination tasks Typed responses to the memory and imagination tasks were coded for internal and external details (Levine et al., 2002). Internal details are episodic information pertaining to a focal memory or imagined event and include details about people, objects, actions, feelings, thoughts, location, and time, among others. External details include semantic information (e.g., "I always loved going to the beach."), reference to repeated events rather than a single episode (e.g., "We went to the beach every Saturday during the summer.") or script-like information (e.g., "First, we would pack a cooler, then put on sunscreen, and finally, find a good spot to catch some rays.").

Coding of the MEPS and memory and imagination tasks was completed by one of three trained raters. Prior to coding participant responses, raters practiced with a set of 15 responses, and high interrater reliability was achieved (standardized Chronbach's alpha $=.94$ for steps, .92 for internal details, and .95 for external details). All raters were naïve to study hypotheses and were blind to diagnosis as well as to which interview had been conducted.

\section{Results}

We conducted a series of mixed-factorial analyses of variance (ANOVAs), with the between-subjects factor of depression status (depressed vs. nondepressed) and the within-subjects factors of interview type (control vs. detailed), step type (relevant vs. other), and detail type (internal vs. external). Subsequent post hoc tests were conducted to determine the nature of observed effects and interactions.

\section{Step generation for problem-solving task}

The results of a mixed-factorial ANOVA revealed a significant Interview Type $\times$ Step Type interaction, $F(1,42)=11.03, p<$ $.005, \eta_{\mathrm{p}}{ }^{2}=.21$. Depression status did not interact with interview type or step type $\left(F_{\mathrm{S}}<2, p \mathrm{~s}>.19\right)$. Critically, depressed participants benefitted from the intervention and generated significantly more relevant steps following the episodic specificity induction $(M=12.4 ; S D=4.0)$ than following the general impressions interview $(M=8.6 ; S D=2.5), t(21)=$ $4.68, p<.001,95 \%$ CI $[-5.51,-2.12], d=1.14$. Consistent with previous work, post hoc analyses indicated that nondepressed participants also generated significantly more relevant steps after completing the episodic specificity induction $(M=$ 13.9; $S D=4.1)$ compared to the general impressions interview $(M=10.2 ; S D=3.7), t(21)=-3.96, p=.001,95 \%$ CI $[-5.68,-$ 1.77], $d=.95$. These results are depicted in Fig. 1. Groups did not differ in the number of other steps following the episodic specificity induction $(p>.4)$. Additionally, there was no difference in either group in the number of other steps generated in response to either interview type, $p \mathrm{~s}>.3$. Data regarding other steps are depicted in Fig. 2. Participants spent more time discussing videos following the encoding specificity induction ( $M=9 \mathrm{mins}, 58 \mathrm{~s} ; S D=3 \mathrm{~min}, 35 \mathrm{~s})$ compared to the general impressions interview $(M=7 \mathrm{mins}, 49 \mathrm{~s} ; S D=3 \mathrm{mins}$, $13 \mathrm{~s}), t(43)=3.77, p<.001, d=0.57$. However, including the difference in time spent between interview formats as a covariate in the above and subsequent analyses did not significantly modify results.

\section{Detail generation for memory and imagination tasks}

Analysis of the memory task revealed a significant Interview Type $\times$ Detail Type interaction, $F(1,42)=30.85, p<.001, \eta_{\mathrm{p}}{ }^{2}=$ .42. Depression status did not interact with either interview type or detail type $(F \mathrm{~s}<2, p \mathrm{~s}>.22)$. Critical to the present study, depressed participants generated significantly more internal memory details following the episodic specificity induction ( $M=23.0 ; S D=10.4)$ compared to the general impressions interview $(M=13.6 ; S D=6.01), t(21)=-5.66, p<.001,95 \%$ CI $[-12.93,-4.98], d=1.11$. Also, as expected, post hoc analyses revealed that nondepressed participants generated significantly more internal details for the memory task following the episodic specificity induction $(M=21.8 ; S D=8.8)$ compared to the general impressions interview $(M=17.0 ; S D=4.2), t(21)=$ $-2.98, p<.01,95 \%$ CI $[-8.10,-1.45], d=.70$. These data are presented in Fig. 1. Groups did not differ in the number of external details for the memory task following either interview type ( $p$ s $>$.70), the data for which are presented in Fig. 2.

Analysis of the imagination task also revealed a significant Interview Type $\times$ Detail Type interaction, $F(1,42)=37.87, p<$ $.001, \eta_{\mathrm{p}}{ }^{2}=.47$. Depression status did not interact with either interview type or detail type $\left(F_{\mathrm{S}}<1\right)$. Post hoc analyses revealed that depressed participants generated significantly more internal details following the episodic specificity induction $(M=21.9 ; S D=8.8)$ compared to the general impressions interview $(M=17.3 ; S D=7.8), t(21)=-4.15, p<.001,95 \%$ CI [-6.90, -2.30], $d=.55$. Nondepressed participants also generated significantly more internal details following the 


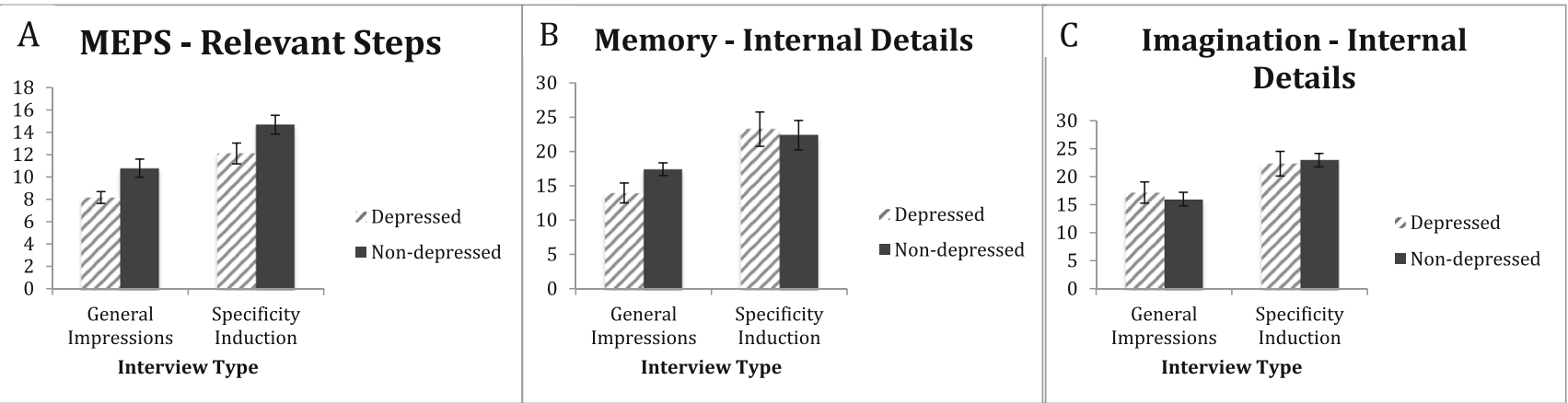

Fig. 1 Mean relevant steps (a) and internal details for memory (b) and imagination (b) tasks as a function of interview type and depression status. Error bars represent $S E$

episodic specificity induction $(M=21.6 ; S D=5.19)$ compared to the general impressions interview $(M=15.9 ; S D=4.9)$, $t(21)=-5.05, p<.001,95 \%$ CI [-8.02, -3.34$], d=1.13$. These data are presented in Fig. 1.There was no difference in the number of external details generated by either group following the episodic specificity induction $(p>.19)$. However, fewer external details were produced following the episodic specificity induction for both the depressed group, $t(21)=2.38, p<$ .05 , and the nondepressed group, $t(21)=3.59, p<.005$. External detail data are presented in Fig. 2.

\section{Correlational analyses of problem solving and memory and imagination performance}

To understand the relations between problem solving, memory, and imagination, we computed averages for relevant steps on the problem-solving task and internal and external details for the memory and imagination tasks. The results of correlational analyses for the depressed group following the episodic specificity induction are presented in Table 1. As depicted in Table 1, the significant positive correlations between internal details and relevant steps, and the lack of an association between external details and relevant steps provide additional support for the involvement of episodic processes in the problem-solving task. However, we do not see such a clear pattern regarding external details and other steps, contrary to previous studies (Madore et al., 2014; Madore \& Schacter, 2014; Sheldon et al., 2015).
No significant correlations between these variables were observed among depressed participants following the general impressions interview. Among nondepressed participants, significant correlations were observed between external details for memory and imagination tasks and other steps. No correlations between relevant steps and any other measures reached significance. Because the same basic pattern was observed when analyzed separately by interview type, we collapsed across interview type. The data for the nondepressed participants are presented in Table 2.

\section{Discussion}

Identifying novel methods of improving problem solving among individuals with depression and incorporating them into existing therapeutic approaches has the potential to advance treatment by reducing problematic symptoms and possibly limiting the duration and frequency of depressive episodes. In the present study, we examined whether an intervention designed to increase access to details in memory (i.e., an episodic specificity induction about a recently watched video; Madore, et al., 2014; Madore \& Schacter, 2014) would lead depressed and nondepressed individuals to access more detailed representations of recalled past and imagined future scenarios and generate significantly more relevant steps on a problem-solving task. As hypothesized, both depressed and

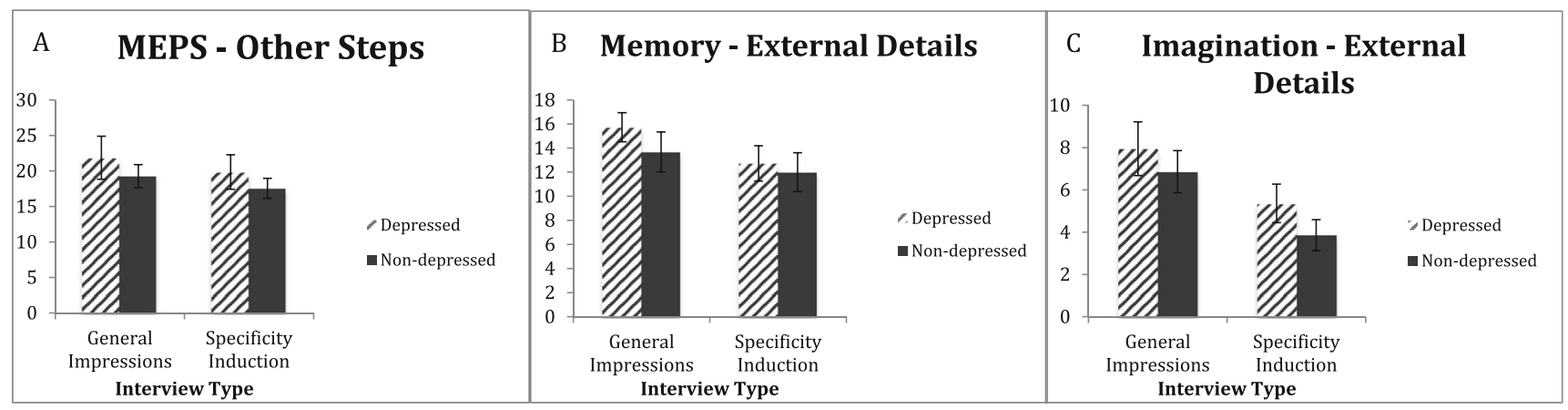

Fig. 2 Mean other steps (a) and external details for memory (b) and imagination (c) tasks as a function of interview type and depression status. Error bars represent $S E$ 
Table 1 Correlations between step types and detail types for depressed participants following the episodic specificity induction

\begin{tabular}{lllllll}
\hline Variable & 1 & 2 & 3 & 4 & 5 & 6 \\
\hline 1. Relevant steps & - & & & & & \\
2. Memory internal details & $.60^{* *}$ & - & & & & \\
3. Imagination internal details & $.40^{+}$ & $.68^{* * *}$ & - & & & \\
4. Other steps & .01 & $.45^{*}$ & .37 & - & & \\
5. Memory external details & -.09 & -.01 & .04 & -.38 & - & \\
6. Imagination external details & .11 & .16 & -.19 & .33 & .11 & - \\
\hline
\end{tabular}

Note. ${ }^{+} p=.06 ; * p<.05 ; * * p<.005 ; * * * p<.001$

nondepressed participants generated more internal details when recalling past personal events and imagining future scenarios following the episodic specificity induction as compared to the control interview. Critically, all participants also generated more relevant steps on the problem-solving task following the episodic specificity induction, which is indicative of improved problem-solving ability. These results suggest that by increasing access to detailed representations of past and future events, it is possible to augment problem solving abilities among people with depression.

The present findings replicate and extend the work of Madore and colleagues (Madore et al., 2014; Madore \& Schacter, 2014) and lend partial support to the constructive episodic simulation hypothesis (Schacter \& Addis, 2007), which posits, among other things, that imagined future scenarios are developed by retrieving and reorganizing information from memory. The flexible use of memories and imagined events can then be applied to the execution of nonmemory tasks, such as the MEPS task used in this study. In support of this notion, our analyses of the performance of depressed participants following the episodic specificity induction revealed a strong positive correlation between the generation of internal details on the memory task and relevant steps and a trend between internal details on the imagination task and relevant steps. These results support our hypothesis that increased episodic detail generation might contribute to enhanced problem solving among individuals with depression

Table 2 Correlations between step types and detail types for nondepressed participants collapsed across interview type

\begin{tabular}{lllllll}
\hline Variable & 1 & 2 & 3 & 4 & 5 & 6 \\
\hline 1. Relevant steps & - & & & & & \\
2. Memory internal details & .28 & - & & & & \\
3. Imagination internal details & .20 & $.42 *$ & - & & & \\
4. Other steps & .01 & .15 & .34 & - & & \\
5. Memory external details & -.13 & $-.51^{*}$ & .10 & $.44^{*}$ & - & \\
6. Imagination external details & -.09 & -.22 & -.34 & $.55^{* *}$ & $.48^{*}$ & - \\
\hline
\end{tabular}

Note. $* p<.05 ; * * p<.01$ and are consistent with the results of previous studies (Madore et al., 2014; Madore \& Schacter, 2014; Sheldon et al., 2015).

Two additional findings are also worth noting. First, it is not obvious what might have contributed to the depressed participants intact ability to generate details for imagined future scenarios, particularly given findings that suggest imagining future scenarios may be more difficult than recalling past events among individuals with depression (Addis et al., 2016). Also, we did not observe the anticipated significant correlations between internal details for the memory and imagination tasks and relevant steps among nondepressed participants. We did, however, observe the expected correlations between external details for memory and imagination tasks and other steps, consistent with previous work. Our results, therefore, diverge somewhat from those of Madore and Schacter (2014), who found internal details for both memory and imagination to be positively correlated with relevant steps among healthy young adults. It is not entirely clear why that relation did not emerge in this study. One possibility is that the nondepressed participants in our study generated numerically fewer internal details on the memory and imagination tasks than participants in the Madore and Schacter study, resulting in a restricted range, which might have limited our ability to detect a relation between these variables. The lack of a correlation between these variables, notwithstanding, the critical finding of this study is that depressed participants demonstrated improved problem solving following the episodic specificity induction, a finding that could have clinical implications.

The mechanism underlying the positive effect of the episodic specificity induction on memory, imagination, and problem solving appears to be that the specificity induction recruits episodic processes that can then support non-memory activities. The episodic specificity induction includes probing questions that encourage participants to adopt a very detail-oriented approach to retrieval. This is in contrast to the control interview, which does not encourage access to specific details, but rather the generation of general thematic information (e.g., people engaged in "kitchen activities"). The results of this and previous research (Madore et al., 2014; Madore \& Schacter, 2014) suggest that the detail-oriented probing questions place participants in a retrieval mode that is biased toward the extraction of more fine-grained information. That high-fidelity retrieval mode is subsequently retained and contributes to improved performance on the memory, imagination, and problemsolving tasks. Thus, the increased access to episodic details engendered by the episodic specificity induction may have allowed for the development of broader, more nuanced perspectives of the scenarios depicted in the problem-solving task, resulting in enhanced availability of or access to relevant steps. This notion that the improved problem-solving performance following the episodic specificity induction hinges upon the retrieval of episodic details is partially supported by the positive correlations observed among depressed participants. Although 
access to semantic memory can often contribute to the execution of complex cognitive tasks (Abraham, 2014; Klein, 2013), the episodic specificity induction employed here does not appear to tap into semantic processing. Nor does the episodic specificity induction simply encourage a more elaborative narrative style, as evidenced in previous studies (Madore et al., 2014; Madore \& Schacter, 2014; Schacter \& Madore, 2016). Further support for this interpretation comes from a recent imaging study that revealed that the episodic specificity induction recruits core networks that are typically associated with episodic processes (Madore, Szpunar, Addis, \& Schacter, 2016).

The intervention used in this study is particularly relevant to depression, which has been linked to deficits in memory (Hach et al., 2014; Söderlund et al., 2014; Williams et al., 2007), imagination (Addis, Hach, \& Tippett, 2016; Holmes, Lange, et al., 2008), and problem solving (Goddard et al., 1996; Raes et al., 2005; Williams et al., 2007). Cognitive deficits in the context of depression appear to be driven, at least in part, by reduced cognitive control (Gotlib \& Joormann, 2010). A related idea is that depressed individuals have difficulty spontaneously engaging in or recruiting strategic search processes (Hertel, 2004), particularly when in open-ended or unstructured circumstances (e.g., the general impressions interview in this study, the MEPS task), and may contribute to reduced access to details of recalled and imagined events (Holmes, Lang, et al., 2008). The structured retrieval mode encouraged by the episodic specificity induction may circumvent the cognitive control difficulties that depressed participants often experience, allowing them to strategically retrieve relevant information, thereby leading to increased generation of episodic details. This interpretation is consistent with recent work by Addis et al. (2016), who reported that diminished strategic retrieval processes in the context of depression contribute to less detailed simulations of future events.

The present findings hint at several potential clinical implications of the episodic specificity induction. Enhancing access to details of personal past events among individuals with depression may contribute to the retrieval of more positive details and attributes and may begin to counteract the negative interpretation bias that is common in depression (Butler \& Mathews, 1983), and therefore lead to a reduction in the experience of depressive symptoms (Neshat-Doost et al. 2013). Similarly, increasing the amount of details that depressed individuals can generate when imagining future scenarios may contribute to increased positive affect (Holmes, Coughtrey, \& Connor, 2008), which may in turn lead to increased behavioral activation and goal pursuit (Libby, Shaeffer, Eibach, \& Slemmer, 2007). Interestingly, recent work suggests that a reduced ability to imagine future scenarios may underlie the development of depressive symptoms and serve to maintain a depressive state once it is established (Roepke \& Seligman, 2016). Thus, increasing access to detailed representations of future events may serve as a protective factor against the development of subsequent depressive episodes. Finally, improving problem-solving performance may limit the duration of depressive episodes and reduce the frequency of suicidal ideation by enhancing the identification and initiation of cognitions and behaviors that may contribute to emotional well-being.

Limitations of the current study include a relatively small sample size. It is possible that the sample size in this study precluded the identification of previously reported correlations between detail generation in memory and imagination tasks and step generation on the problem-solving task (Madore et al., 2014; Madore \& Schacter, 2014; Sheldon et al., 2015). However, the anticipated pattern of correlations was recently obtained in a smaller sample than that of the current study (Sheldon et al., 2015), calling this interpretation into question. Additionally, although Anderson, Goddard, and Powell (2011) revealed that MEPS performance was a significant predictor of future depressive symptoms, the ecological validity of the MEPS has been questioned (Anderson, Goddard, \& Powell, 2009). Finally, the inclusion of a measure of fluency may have been informative to further clarify, in this particular study, the role of strategic retrieval efforts.

Limitations notwithstanding, the results of the current study reveal that a brief episodic specificity induction can lead to improved social problem solving among individuals with depression. The simplicity and brevity of this intervention may make it a versatile therapeutic tool that could be applied in a wide range of clinical settings. A number of future directions for research are worthy of pursuit. First, it will be important to identify ways of increasing the duration of this effect, as Jing, Madore, and Schacter (2016) recently reported that the effect is short-lived. One way to extend the effect may be to engage individuals in brief, intermittent sessions in which individuals are asked to complete simulation exercises. Once a means of extending the effect has been established, longer-term intervention studies could investigate the feasibility and therapeutic impact of incorporating brief, self-directed exercises into a daily routine. Future work could also begin to investigate the effectiveness of this intervention when applied to personally relevant, emotion-laden problems such as those often encountered in the context of depression, similar to the work of Jing et al. (2016) in the context of worry.

\section{References}

Abraham, A. (2014). Creative thinking as orchestrated by semantic processing vs. cognitive control brain networks. Frontiers in Human Neuroscience, 8(95), 1-6.

Addis, D. R., Hach, S., \& Tippett, L. J. (2016). Do strategic processes contribute to the specificity of future simulation in depression? British Journal of Clinical Psychology, 55(2), 167-186.

Anderson, R. J., Goddard, L., \& Powell, J. H. (2009). Social problemsolving processes and mood in college students: An examination of self-report and performance-based approaches. Cognitive Therapy and Research, 33(2), 175-186.

Anderson, R. J., Goddard, L., \& Powell, J. H. (2011). Social problemsolving and depressive symptom vulnerability: The importance of 
real-life problem-solving performance. Cognitive Therapy and Research, 35, 48-56.

Butler, G., \& Mathews, A. (1983). Cognitive processes in anxiety. Advances in Behaviour Research and Therapy, 5, 51-62.

Cannon, B., Mulroy, R., Otto, M. W., Rosenbaum, J. F., Fava, M., \& Neirenberg, A. (1999). Dysfunctional attitudes and poor problem solving skills predict hopelessness in major depression. Journal of Affective Disorders, 55, 45-49.

Conway, M. A., \& Pleydell-Pearce, C. W. (2000). The construction of autobiographical memories in the self-memory system. Psychological Review, 107(2), 261-288.

First, M. B., Spitzer, R. L., Gibbons, M., \& Williams, J. B. W. (2002). Structured clinical interview for DSM-IV-TR axis I disorders (Research version, nonpatient ed., SCID-I/NP). New York: New York State Psychiatric Institute, Biometrics Research.

Goddard, L., Dritschel, B., \& Burton, A. (1996). Role of autobiographical memory in social problem solving and depression. Journal of Abnormal Psychology, 105, 609-616.

Gotlib, I. H., \& Joormann, J. (2010). Cognition and depression: Current status and future directions. Annual Review of Clinical Psychology, 6, 285-312.

Hach, S., Tippett, L. J., \& Addis, D. R. (2014). Neural changes associated with the generation of specific past and future events in depression. Neuropsychologia, 65, 41-55.

Hertel, P. T. (2004). Memory for emotional and non-emotional information in depression: A question of habit? In D. Reisberg \& P. T. Hertel (Eds.), Memory and emotion (pp. 186-216). New York: Oxford University Press.

Holmes, E. A., Coughtrey, A. E., \& Connor, A. (2008). Looking at or through rose-tinted glasses? Imagery perspective and positive mood. Emotion, 8(6), 875-879.

Holmes, E. A., Lange, T. J., Moulds, M. L., \& Steele, A. M. (2008). Prospective and positive mental imagery deficits in dysphoria. Behaviour Research and Therapy, 46, 976-981.

Irish, M., \& Piquet, O. (2013). The pivotal role of semantic memory in remembering the past and imagining the future. Frontiers in Behavioral Neuroscience, 7(27), 1-11.

Jing, H. G., Madore, K. P., \& Schacter, D. L. (2016). Worrying about the future: An episodic specificity induction impacts problem solving, reappraisal, and well-being. Journal of Experimental Psychology: General, 145(4), 402-418.

Klein, S. B. (2013). The temporal orientation of memory: It's time of a change of direction. Journal of Applied Research in Memory and Cognition, 2, 222-234.

Klein, S. B., \& Gangi, C. E. (2010). The multiplicity of self: Neuropsychological evidence and its implications for the self as a construct in psychological research. Annals of the New York Academy of Sciences, 1191, 1-15.

Levine, B., Svoboda, E., Hay, J. F., Winocur, G., \& Moscovitch, M. (2002). Aging and autobiographical memory: Dissociating episodic from semantic retrieval. Psychology and Aging, 17, 677-689.

Libby, L. K., Shaeffer, E. M., Eibach, R. P., \& Slemmer, J. A. (2007). Picture yourself at the polls-Visual perspective in mental imagery affects self-perception and behavior. Psychological Science, 18(3), 199-203.

Linda, W. P., Marroquín, B., \& Miranda, R. (2012). Active and passive problem solving as moderators of the relation between negative life event stress and suicidal ideation among suicide attempters and nonattempters. Archives of Suicide Research, 16, 183-197.

Madore, K. P., Gaesser, B., \& Schacter, D. L. (2014). Constructive episodic simulation: Dissociable effects of a specificity induction on remembering, imagining, and describing in young and older adults. Journal of Experimental Psychology: Learning, Memory, \& Cognition, 40(3), 609-622.
Madore, K. P., \& Schacter, D. L. (2014). An episodic specificity induction enhances means ends problem solving in young and older adults. Psychology and Aging, 29(4), 913-924.

Madore, K. P., Szpunar, K. K., Addis, D. R., \& Schacter, D. L. (2016). Episodic specificity induction impacts activity in a core brain network during construction of imagined future experiences. Proceedings of the National Academy of Sciences, 113(38), 10696-10701.

Neshat-Doost, H. T., Dalgleish, T., Yule, W., Kalantari, M., Ahmadi, S. J., Dyregrov, A., \& Jobson, L. (2013). Enhancing autobiographical memory specificity through cognitive training: An intervention for depression translated from basic science. Clinical Psychological Science, 1, 84-92.

Platt, J., \& Spivack, G. (1975). Manual for the means-end problem-solving test (MEPS): A measure of interpersonal problem solving skill. Philadelphia: Hahnemann Medical College and Hospital.

Raes, F., Hermans, D., Williams, J. M. G., Demyttenaere, K., Sabbe, B., Pieters, G., \& Eelen, P. (2005). Reduced specificity of autobiographical memory: A mediator between rumination and ineffective social problem-solving in major depression? Journal of Affective Disorders, 87, 331-335.

Roepke, A. M. \& Seligman, M. E. P. (2016). Depression and prospection. British Journal of Clinical Psychology, 55, 23-48.

Schacter, D. L. (2012). Adaptive constructive processes and the future of memory. American Psychologist, 67, 603-613.

Schacter, D. L., \& Addis, D. R. (2007). The cognitive neuroscience of constructive memory: Remembering the past and imagining the future. Philosophical Transactions of the Royal Society of London Series B, Biological Sciences, 362, 773-786.

Schacter, D. L. \& Madore, K. P.(2016) Remembering the past and imagining the future: Selective effects of an episodic specificity induction on detail generation. The Quarterly Journal of Experimental Psychology, 69(2), 285-298.

Schacter, D. L., Addis, D. R., \& Buckner, R. L. (2008). Episodic simulation of future events: Concepts, data, and applications. Annals of the New York Academy of Sciences, 1124, 39-60.

Sheldon, S., McAndrews, M. P., \& Moscovitch, M. (2011). Episodic memory processes mediated by the medial temporal lobes contribute to open-ended problem solving. Neuropsychologia, 49, 2439-2447.

Sheldon, S., Vandermorris, S., Al-Haj, M., Cohen, S., Winocur, G., \& Moscovitch, M. (2015). Ill-defined problem solving in amnestic mild cognitive impairment: Linking episodic memory to effective solution generation. Neuropsychologia, 68, 168-175.

Söderlund, H., Moscovitch, M., Kumar, N., Daskalakis, Z. J., Flint, A., Herrmann, N., \& Levine, B. (2014). Autobiographical memory in major depressive disorder. Journal of Abnormal Psychology, 123(1), $51-60$.

Szpunar, K. K. (2010). Episodic future thought: An emerging concept. Perspectives on Psychological Science, 5, 142-162.

Tulving, E. (1983). Elements of episodic memory. New York: Oxford University Press.

Tulving, E. (1985). Memory and consciousness. Canadian Journal of Psychology, 26, 1-12.

Vandermorris, S., Sheldon, S., Winocur, G., \& Moscovitch, M. (2013). Differential contributions of executive and episodic memory functions to problem solving in younger and older adults. Journal of the International Neuropsychological Society, 19, 1087-1096.

Williams, J. M. G., Barnhofer, T., Crane, C., Herman, D., Raes, F., Watkins, E., \& Dalgleish, T. (2007). Autobiographical memory specificity and emotional disorder. Psychological Bulletin, 133(1), 122-148.

Williams, J. M. G., Barnhofer, T., Crane, C., \& Duggan, D. S. (2006). The role of overgeneral memory in suicidality. Cognition and suicide: Theory, research, and therapy (pp. 173-192). Washington, DC: American Psychological Association. doi:10.1037/11377-008 